\title{
Serum dioxin and DNA methylation in the sperm of operation ranch hand veterans exposed to Agent Orange
}

\author{
Karl T. Kelsey ${ }^{1,2^{*}+} \mathbb{D}$, Matthew Rytel ${ }^{1+}$, Edward Dere ${ }^{2}$, Rondi Butler ${ }^{1,2}$, Melissa Eliot ${ }^{1}$, Susan M. Huse ${ }^{3}$,
}

E. Andres Houseman ${ }^{4}$, Devin C. Koestler ${ }^{5}$ and Kim Boekelheide ${ }^{2}$

\begin{abstract}
Background: Exposure to the herbicide Agent Orange during the Vietnam War was widespread and is associated with numerous adverse health outcomes. A continuing concern of veterans is the possibility that exposure to the dioxin-containing herbicide might induce adverse reproductive outcomes. We sought to assess whether exposure to Agent Orange in Vietnam was associated with changes in DNA methylation in sperm in a subset of Vietnam veterans who participated in the Air Force Health Study (AFHS).
\end{abstract}

Methods: We studied 37 members of the AFHS chosen to have no, low, medium or high exposure to Agent Orange, based upon serum dioxin levels obtained during a series of examinations. DNA from stored semen was extracted and DNA methylation assessed on the Illumina $450 \mathrm{~K}$ platform.

Results: Initial epigenome-wide analysis returned no loci that survived control for false discovery. However, the TEAD3 gene had four different $\mathrm{CPG}$ sites that showed loss of DNA methylation associated with dioxin exposure. Analysis assessing regional DNA methylation changes revealed 36 gene regions, including the region of the imprinted gene H19 to have altered DNA methylation associated with high exposure compared to the low exposure group. Additional comparison of our data with sperm DNA methylation data from Russian boys exposed to dioxin found an additional 5 loci that were altered in both studies and exhibited a consistent direction of association.

Conclusions: Studying a small number of sperm samples from veterans enrolled in the AFHS, we did not find evidence of significant epigenome-wide alterations associated with exposure to Agent Orange. However, additional analysis showed that the $\mathrm{H} 19$ gene region is altered in the sperm of Agent Orange-exposed Ranch Hand veterans. Our study also replicated several findings of a prior study of dioxin-exposed Russian boys. These results provide additional candidate loci for further investigation and may have implications for the reproductive health of dioxin-exposed individuals.

Keywords: Sperm, Dioxin, Agent Orange, DNA methylation, Epigenetics

\section{Introduction}

Agent Orange was an herbicide/defoliant that was sprayed across Vietnam and Southeast Asia by the U.S. Air Force during the war in Vietnam as part of a mission termed Operation Ranch Hand. The intent of this widespread use

\footnotetext{
* Correspondence: Karl_Kelsey@brown.edu

${ }^{\dagger}$ Karl T. Kelsey and Matthew Rytel are co-first authors.

${ }^{1}$ Department of Epidemiology, Brown University School of Public Health,

Providence, RI 02912, USA

${ }^{2}$ Department of Pathology and Laboratory Medicine, Brown University

School of Public Health, Providence, RI 02912, USA

Full list of author information is available at the end of the article
}

was to clear vegetative cover used by the enemy as well as to destroy crops that fed them. The most toxic form of dioxin - 2,3,7,8-tetrachlorodibenzo- $p$-dioxin (TCDD) - was present in Agent Orange (AO) as a byproduct of the industrial processes employed to manufacture the herbicide. The soldiers who were tasked with receiving, storing, distributing, mixing and applying Agent Orange (Ranch Hands) were heavily exposed to it, and thus at risk for health issues associated with exposure to TCDD. Dioxins in general, and TCDD in particular, are extremely lipophilic and thus persistent in both the individual and the

(c) The Author(s). 2019 Open Access This article is distributed under the terms of the Creative Commons Attribution 4.0 International License (http://creativecommons.org/licenses/by/4.0/), which permits unrestricted use, distribution, and reproduction in any medium, provided you give appropriate credit to the original author(s) and the source, provide a link to the Creative Commons license, and indicate if changes were made. The Creative Commons Public Domain Dedication waiver (http://creativecommons.org/publicdomain/zero/1.0/) applies to the data made available in this article, unless otherwise stated. 
environment. The half-life of TCDD in the human body has been estimated to range from 7 to 11 years $[1,2]$.

The health effects of exposure to AO have been assessed and reviewed by the National Academy of Sciences on an ongoing basis since 1994 [1]. The range of adverse health effects that have been associated with $\mathrm{AO}$ exposure is broad, including likely associations with soft-tissue sarcoma, non-Hodgkin lymphoma, chronic lymphocytic leukemia, Hodgkin lymphoma, chloracne, hypertension and monoclonal gammopathy of undetermined significance [2]. Controversy exists around the nature of any association between exposure to $\mathrm{AO}$ and subsequent $\mathrm{F} 1$ birth defects, but the most recent review concludes that "evidence remains inadequate or insufficient to determine whether there is an association between exposure to [agent orange] and birth defects in the children of Vietnam veterans" [2]. While the efforts to assess possible health effects have been intense, it is apparent that little data has been available to address the significant emerging concerns of Vietnam veterans around the possible health effects of paternal $\mathrm{AO}$ exposures on their descendants. To our knowledge, there are no data on $\mathrm{AO}$ exposure and subsequent reproductive outcomes or transgenerational health effects in Vietnam veterans specifically, and as the Veterans Agent Orange Committee notes in the most recent report "more work in this area is warranted"; it recommends "further specific study of the health of offspring of male Vietnam veterans" [2].

TCDD is a well-known endocrine disrupting chemical [3] that has recently been shown to alter the epigenome [4]. Considerable in-vitro evidence has demonstrated that TCDD is particularly epigenetically active in developing organisms [5-9] as well as in male germ cells (reviewed in [10]). Further, recent studies report that dioxin exposure (as well as other endocrine disrupting agents) can induce inter- and transgenerational alterations in sperm DNA methylation [11-14]. Exposure to TCDD also has been associated with poor semen parameters in exposed peripubertal boys [15]. Similar findings have been reported after childhood exposures to high levels of TCDD [16]. Recently, studying a cohort of Russian boys exposed to TCDD, Pilsner et al. [17] reported 666 CpGs to be aberrantly methylated in the sperm epigenome in the groups with the highest compared to the lowest serum TCDD concentrations.

Given the growing literature showing the potential for TCDD exposure to alter the germline epigenome, we have studied samples from veterans involved in Operation Ranch Hand. Intense interest in the possible health effects of exposure to AO in Vietnam prompted the Air Force, in 1982, to launch a study of the soldiers tasked with handling herbicides and defoliants in Vietnam, called the Air Force Health Study (AFHS), with an aim to determine the health effects these soldiers might experience as a consequence of their exposure. In 1996 Henriksen et al [18] reported on the reproductive health of these veterans and observed no testicular abnormalities, no alterations in sperm count or percentage of abnormal sperm in the AFHS associated with serum dioxin levels. However, as these measures are known to be poorly predictive of adverse reproductive outcomes, we focused upon the possible impacts of TCDD on the sperm epigenome, targeting our investigation on the induction of epigenetic changes in a subset of the Ranch Hand veterans. Additionally, with increasing evidence in the literature of dioxin-induced transgenerational alterations to the sperm epigenome, greater knowledge of the epigenetic changes that occurred in veterans exposed to Agent Orange is essential to understanding the potential impacts on their descendants.

\section{Methods}

The Air Force Health Study is a prospective study that began in 1982, in which consenting veterans, over numerous visits (study 'cycles') received physical examinations, health questionnaires, medical record reviews, and biospecimen sampling [19]. The study included examinations that occurred in cycles at time points of $1,3,5,10,15$, and 20 years after the initiation of the study [19]. Semen was collected from the entire AFHS cohort in 1982, and subsequently, serum dioxin assays were performed by the Centers for Disease Control on 777 Ranch Hands and 1174 control subjects during cycles 3 to $6(1987-2002)$ of the study [20]. We received semen samples from cycles 2,5 and 6, and isolated genomic DNA. The process of isolation of the semen has been described by the Vietnam Experience Study [21]. While somatic cells are not known to have been assessed in these samples, contamination will not be in excess of approximately 1\% [22] and thus this is unlikely to affect the data. Genome-wide DNA methylation levels were assessed from the control group (nonRanch Hand) and Ranch Hand personnel using the Illumina $450 \mathrm{~K}$ Infinium Methylation Beadchips. The raw data from the beadchips was processed and normalized, and met all quality control requirements. De-identified personnel information was used to determine potential cofounders, including age, smoking history, and body mass index (BMI). Exposure categories - defined according to lipid-adjusted serum levels of dioxin - were characterized as control (non-Ranch Hand control group with dioxin adjusted serum levels ranging from 2.21 to 7.05 ppt; $n=12$ ), low (dioxin adjusted serum levels 5.6 to 8.0 ppt; $n=4$ ), moderate (dioxin adjusted serum levels between 8.9 to $19.6 \mathrm{ppt} ; n=11$ ); and high (dioxin adjusted serum levels 26.6 to $167.6 \mathrm{ppt} ; n=15$ ). There were no participants with non-detectable dioxin levels. Subjects in the control group were stationed in Southeast Asia during the 
Vietnam War, and therefore were not exposed to Agent Orange [23].

Using the Infinium $450 \mathrm{~K}$ platform, we obtained DNA methylation measurements for each specimen; on this platform, DNA methylation is typically measured on an "average beta" scale, which represents the fraction of methylated molecules (for each target site) and is bounded by 0 (unmethylated) and 1 (methylated). We performed standard normalization and QA/QC procedures, including the removal of poor quality $\mathrm{CpGs}$ and samples on the basis of detection $p$-value [24] removal of cross-reactive CpGs [25], and the removal of CpGs containing polymorphic sites with a minor allele frequency $>1 \%$ at the target site or within the probe sequence. Background correction and dye-bias normalization was performed using the Noob method [26] contained in the minfi Bioconductor package. Between array-normalization was performed using the FunNorm procedure [27], which removes unwanted technical variation by regressing out variability explained by the control probes present on the array. In addition to background-correcting and normalizing the raw values, we performed BMIQ adjustment to correct for probe design bias [28] and subsequently used the ComBat procedure to adjust for batch effects. Altogether, the starting number of $\mathrm{CpG}$ loci was reduced from 485,577 to 428,936 loci.

When analyzing the association of serum dioxin level with methylation in the sperm tissue, dioxin level was modeled three ways: as a categorical variable (control vs. high), as a continuous variable using natural logtransformed dioxin, and as trend of dioxin $(0=$ control, $0.33=$ low, $0.67=$ medium, $1=$ high). We used a linear model fit with an empirical Bayes method that adjusted for age, BMI, smoking, and dioxin levels, to determine the levels of methylation.

Data were initially analyzed using an epigenome wide association study (EWAS) approach to identify potential differentially methylated CpGs between control and high-dioxin-exposed subjects. To do this, surrogate variable analysis (SVA) was used to additionally remove any batch effects [29]. Employing the SVA package, we estimated the optimal number of surrogate variables was 5 . Subsequently, linear models with empirical Bayes estimation adjusting for age, smoking, BMI, and the five surrogate variables were run. The Benjamini-Hochberg method was utilized to control the false discovery rate.

In order to select CpGs from the $450 \mathrm{~K}$ array that align with those from the Pilsner et al. study [17], which used reduced representation bisulfite sequencing, we employed the UCSC Genome Browser liftOver tool (https://genome.ucsc.edu/cgi-bin/hgLiftOver). Of the 666 loci found to be significantly associated with dioxin exposure, we were able to match 37 to our $450 \mathrm{~K}$ data.
We repeated the SVA analysis on this subset of data, again using five surrogate variables.

Finally, we looked for effects within regions of DNA, rather than individual CpGs. DMRcate (version 1.18.0), which has previously been shown to perform favorably as compared competing methods for DNA methylation based regional-level analyses [30], was employed here to identify differentially methylated regions associated with serum dioxin levels. We used betas for all CpGs that were not within SNPs and ran DMRcate to calculate the average change for each region in beta values for control subjects compared to high exposure subjects, as well as both the Stouffer $p$-value [31] for regional-level statistical significance and the minimum adjusted $\mathrm{p}$-value from the CpGs constituting the identified regions. We then sorted the 46,470 regions by the minimum adjusted p-value from the CpGs constituting the identified regions to identify putative regions with differing patterns of DNA methylation as a function of exposure level.

\section{Results}

We obtained sperm methylation data for 37 members of the AFHS. Demographic data available from the participants included smoking status (ever vs. never), BMI, and age. We stratified the participants by serum dioxin levels (see Methods). Eleven of the veterans were classified as not exposed to Agent Orange and had a mean serum dioxin concentration of $4.3 \mathrm{pg} / \mathrm{g}$ lipid; four were exposed to low levels with a mean serum dioxin concentration of $6.5 \mathrm{pg} / \mathrm{g}$ lipid; eleven were exposed to medium levels with a mean serum dioxin concentration of $13.4 \mathrm{pg} / \mathrm{g}$ lipid; and eleven were exposed to high levels with a mean serum dioxin concentration of $61.1 \mathrm{pg} / \mathrm{g}$ lipid. The only significant difference between the four groups was the serum concentration of TCDD (Table 1).

Our initial agnostic approach to this data consisted of an epigenome-wide analysis, comparing the high exposed group with the low exposed. There were no CpGs with $\mathrm{q}<0.05$ after multiple testing adjustment, however several loci showed relatively large differences in methylation values between the highly exposed and the controls (Table 2). Of particular interest is the TEAD3 gene where four different CpG sites showed similar loss of DNA methylation associated with dioxin exposure. The volcano plot resulting from a comparison of CpG-specific methylation values and dioxin exposure is shown in Additional file 1: Figure S1 while the heatmap of the unsupervised clustering analysis is shown in Fig. 1.

Linear models were run with empirical Bayes estimates adjusting for age, smoking, BMI, and five surrogate variables, with log-transformed dioxin as the predictor. All 37 subjects were included in this analysis. We found associations of methylation with TCDD levels in 24,548 
Table 1 Demographics of the study population stratified by dioxin level

\begin{tabular}{llllll}
\hline & Control $(N=11)$ & Low $(N=4)$ & Medium $(N=11)$ & High $(N=11)$ & All $(N=37)$ \\
\hline Age [Mean (SD)] & $70.7(6.8)$ & $79.5(10.4)$ & $77.0(8.5)$ & $68.9(4.1)$ & $73.0(7.9)$ \\
Smoking: Never [N (\%)] & $2(18.2)$ & $1(25.0)$ & $3(27.3)$ & $4(36.4)$ & $10(27.0)$ \\
Smoking: Former [N (\%)] & $8(72.7)$ & $3(75.0)$ & $7(63.6)$ & $5(45.5)$ & $23(62.2)$ \\
Smoking: Current [N (\%)] & $1(9.1)$ & $0(0.0)$ & $1(9.1)$ & $2(18.2)$ & $4(10.8)$ \\
BMI [Mean (SD)] & $32.4(5.7)$ & $25.7(1.8)$ & $30.9(3.7)$ & $29.6(3.6)$ & $30.4(4.5)$ \\
Dioxin [Mean (SD), Range] (ppt) & $4.3(1.4), 2.2-7.1$ & $6.5(0.8), 5.6-7.2$ & $13.4(3.3), 8.9-19.6$ & $61.1(46.2), 26.6-167.6$ & $24.1(34.7), 2.2-167.6$ \\
\hline
\end{tabular}

of 428,936 loci $(5.7 \%)$, but none were statistically significant at the 5\% FDR level. The majority of loci were positively associated with dioxin; that is, higher levels of dioxin were associated with higher levels of methylation. Effect levels ranged from -0.23 to 0.17 change in methylation beta-values per unit increase in log dioxin.

Since our power to detect epigenome-wide differences in DNA methylation associated with Agent Orange exposure was quite low, a candidate-gene approach was also conducted, comparing our data with that of Pilsner et al [17], who studied sperm of dioxin-exposed Russian boys. The Ranch Hand methylation data was directly compared to that of Russian Children's Study after extracting overlapping loci. Since the Russian Children were studied using Reduced Representation Bisulfite Sequencing (RRBS) only 37 of the 666 CpGs reported to be associated with dioxin exposure in that report were available for analysis. After completing a t-test for the Ranch Hand data at each of the 37 overlapping loci, we found five to have a significant difference in methylation $(p<0.05)$ between control and high exposure groups, and two additional loci to be marginally significant before correction for multiple comparisons $(p<0.1$; Table 3). After FDR correction none of the loci were statistically significant (Table 3). Several genes contained more than one of the 37 overlapping CpGs, with SAMD11 housing the most $(N=4)$ followed by PGBD5 $(N=3)$. Other genes that contained multiple overlapping CpGs with the Russian Children's Study include KCNN1, $F A M 83 C$, and RBM33. To test for the directionality of the methylation at each of these sites, we compared the difference in methylation beta values between the high and low exposure groups (Table 3 ). Interestingly, the 3 sites associated with the PGBD5 gene, when compared by dioxin exposure level in the Ranch Hand participants with the data from the Russian boys [17], were all statistically significantly altered before FDR correction and showed common directionality. The MBP locus showed evidence of significant demethylation and the two sites in the KCNN1 locus were borderline significantly altered, and in each case the change was in the same direction as reported in Pilsner et al [17]. The remaining sites were either not significantly altered or showed inconsistent directional change when compared with the
Pilsner et al [17] data. We also examined the AhR pathway, since dioxin is known to interact with genes in this pathway, for alterations in methylation of these loci that correlated with TCDD level but did not find any of statistical significance (data not shown).

Due to the low power to find significant associations in $\mathrm{CpG}$ by $\mathrm{CpG}$ analyses, DMRcate software was also employed to similarly examine coordinate alterations in gene regions that associate with TCDD level. While none of the of the 46,470 regions examined resulted a statistically significant Stouffer $p$-value after adjustment for multiple testing, we did observe 31 regions with minimum adjusted $\mathrm{p}$-value $<10^{-6}$ from the CpGs constituting the region. Of these regions, 5 were located in chromosome 11 , including a region with a p-value of $<10^{-28}$ which is associated with the imprinted gene H19 and contains 50 CpGs. Most of the regions (26 out of 31) showed hypomethylation for high dioxin subjects compared to controls (Fig. 2), including the most significant region for which the average beta value was 0.15 lower (on the beta-value scale) for highly exposed subjects compared to controls. Interestingly, the variation in DNA methylation was most evident at the site of the imprinting control region (Fig. 2). The complete list of regions where altered patterns of DNA methylation were significantly associated with $\mathrm{AO}$ exposure are shown in Table 4.

\section{Discussion}

We have directly investigated the association of exposure to the dioxin-containing herbicide toxicant Agent Orange, widely used during the Vietnam War, with demonstrable alterations in the epigenome of sperm. AO was highly contaminated with TCDD, which is known to be an endocrine disrupting chemical, and evidence is accumulating that it is associated with abnormal reproductive health endpoints. Our study of a small number of AO-exposed Operation Ranch Hand veterans enrolled in the Air Force Health Study found no evidence of any statistically significant exposure-associated alterations in DNA methylation when studied genome-wide in approximately 450,000 loci. However, there were genes, such as TEAD3, with multiple loci seemingly coordinately altered in the DNA from sperm in the exposed 
Table 2 Top 50 EWAS loci ranked by the change in beta value comparing highest exposed with control

\begin{tabular}{|c|c|c|c|c|c|c|c|}
\hline Gene & CpG & $\mathrm{CHR}$ & Distance to TSS & Change in beta value & $P$-value & Mean beta in high expos & Mean beta in low expos \\
\hline UMODL1 & cg08880261 & 21 & 66 & -0.6 & 5.00E-04 & 0.24 & 0.53 \\
\hline NLRC5 & cg03146649 & 16 & 175 & -0.36 & 0.0013 & 0.6 & 0.8 \\
\hline \multirow[t]{2}{*}{$C C L 3 \angle 3, C C L 3 L 1$} & cg03071134 & 17 & 2255 & 0.35 & 0.001 & 0.87 & 0.65 \\
\hline & cg16316180 & 10 & 3767 & -0.31 & 4.00E-04 & 0.43 & 0.63 \\
\hline \multirow[t]{3}{*}{ SERPINA6 } & cg11054397 & 14 & 2039 & 0.3 & $1.00 \mathrm{E}-04$ & 0.98 & 0.8 \\
\hline & $\operatorname{cg} 25345625$ & 10 & 3889 & -0.28 & $8.00 \mathrm{E}-04$ & 0.34 & 0.51 \\
\hline & cg20710519 & 5 & 267 & -0.26 & 0.0011 & 0.32 & 0.61 \\
\hline NRBP2 & cg00061150 & 8 & 380 & -0.26 & 0.0013 & 0.61 & 0.77 \\
\hline TRPV2 & cg04702538 & 17 & 117 & 0.25 & $2.00 \mathrm{E}-04$ & 0.93 & 0.85 \\
\hline NRBP2 & $\operatorname{cg} 24023418$ & 8 & 534 & -0.24 & $6.00 \mathrm{E}-04$ & 0.68 & 0.84 \\
\hline$K D M 2 B$ & cg04682193 & 12 & 354 & -0.24 & 0.0012 & 0.76 & 0.9 \\
\hline SPAM1 & cg04471202 & 7 & 906 & 0.23 & $6.00 \mathrm{E}-04$ & 0.31 & 0.17 \\
\hline \multirow[t]{2}{*}{ ERICHI } & cg02209998 & 8 & 384 & 0.22 & 4.00E-04 & 0.8 & 0.7 \\
\hline & cg04038019 & 20 & 501 & -0.21 & 5.00E-04 & 0.71 & 0.84 \\
\hline PTPRN2 & cg23887345 & 7 & 14,040 & -0.21 & 8.00E-04 & 0.26 & 0.46 \\
\hline C9orf5 & cg14364129 & 9 & 940 & -0.2 & $9.00 \mathrm{E}-04$ & 0.7 & 0.78 \\
\hline TEAD3 & cg14909614 & 6 & 694 & -0.2 & 0.0012 & 0.19 & 0.31 \\
\hline NRBP2 & cg12515402 & 8 & 653 & -0.19 & 4.00E-04 & 0.64 & 0.75 \\
\hline \multirow[t]{3}{*}{ ARHGEF10 } & cg06807837 & 8 & 412 & -0.19 & $1.00 \mathrm{E}-04$ & 0.35 & 0.54 \\
\hline & cg14635160 & 9 & 3896 & 0.19 & 0.0014 & 0.25 & 0.12 \\
\hline & cg19356629 & 10 & 3037 & -0.19 & 7.00E-04 & 0.74 & 0.86 \\
\hline CCL3 & cg22518733 & 17 & 1973 & -0.19 & 8.00E-04 & 0.57 & 0.66 \\
\hline \multirow[t]{2}{*}{ ARHGEF10 } & cg07694735 & 8 & 348 & -0.18 & $9.00 \mathrm{E}-04$ & 0.32 & 0.5 \\
\hline & cg23154021 & 12 & 174 & -0.18 & 0.0011 & 0.41 & 0.61 \\
\hline \multirow[t]{2}{*}{ TEAD3 } & cg22424903 & 6 & 1059 & -0.18 & $6.00 \mathrm{E}-04$ & 0.11 & 0.22 \\
\hline & cg08576350 & 20 & 608 & -0.17 & 4.00E-04 & 0.76 & 0.89 \\
\hline \multirow[t]{2}{*}{ TEAD3 } & cg01487910 & 6 & 563 & -0.17 & $6.00 \mathrm{E}-04$ & 0.14 & 0.24 \\
\hline & cg14181345 & 9 & 1634 & -0.17 & $3.46 \mathrm{E}-05$ & 0.65 & 0.72 \\
\hline CADPS2 & cg23390958 & 7 & 315 & 0.16 & 3.00E-04 & 0.99 & 0.92 \\
\hline AJAP1 & cg21159274 & 1 & 808 & -0.15 & 0.0011 & 0.42 & 0.52 \\
\hline ACCN1 & cg15744026 & 17 & 1045 & 0.14 & 0.001 & 0.53 & 0.51 \\
\hline TEAD3 & cg01546820 & 6 & 263 & -0.13 & $5.00 \mathrm{E}-04$ & 0.08 & 0.16 \\
\hline WDR27 & cg00392003 & 6 & 2873 & -0.12 & 4.00E-04 & 0.6 & 0.68 \\
\hline \multirow[t]{2}{*}{ EPAS1 } & cg09119656 & 2 & 533 & 0.12 & $2.00 \mathrm{E}-04$ & 0.99 & 0.94 \\
\hline & cg27511894 & 1 & 544 & 0.12 & 0.0014 & 0.82 & 0.76 \\
\hline \multirow[t]{2}{*}{ CLEC4E } & cg12762799 & 12 & 410 & -0.12 & $6.00 \mathrm{E}-04$ & 0.39 & 0.45 \\
\hline & cg05240760 & 2 & 75,792 & 0.11 & 7.00E-04 & 0.78 & 0.78 \\
\hline ATP10A & cg07986058 & 15 & 26 & -0.11 & 0.0011 & 0.34 & 0.41 \\
\hline \multirow[t]{2}{*}{ EPB41L3 } & cg01673082 & 18 & 2874 & -0.1 & 4.70E-05 & 0.14 & 0.21 \\
\hline & cg06654337 & 6 & 12,717 & -0.6 & 5.00E-04 & 0.15 & 0.16 \\
\hline WTIP & cg05620261 & 19 & 804 & -0.36 & 0.001 & 0.02 & 0.12 \\
\hline FAM120A & cg15886017 & 9 & 322 & 0.35 & 5.77E-05 & 0.77 & 0.83 \\
\hline$D H R S 7 B$ & cg06582708 & 17 & 945 & -0.31 & 4.00E-04 & 0.33 & 0.31 \\
\hline C4orf42 & cg21793579 & 4 & 1100 & 0.3 & 1.00E-04 & 0.28 & 0.28 \\
\hline
\end{tabular}


Table 2 Top 50 EWAS loci ranked by the change in beta value comparing highest exposed with control (Continued)

\begin{tabular}{llllllll}
\hline Gene & CpG & CHR & Distance to TSS & Change in beta value & $P$-value & Mean beta in high expos & Mean beta in low expos \\
\hline RNASE1 & cg05958352 & 14 & 789 & -0.28 & $8.00 \mathrm{E}-04$ & 0.69 & 0.76 \\
NTNG1 & cg14787581 & 1 & 1367 & -0.26 & 0.0011 & 0.79 & 0.76 \\
& cg12951997 & 12 & 1369 & -0.26 & 0.0013 & 0.88 & 0.91 \\
TUBB8 & cg09677976 & 10 & 1317 & 0.25 & $2.00 \mathrm{E}-04$ & 0.03 & 0.05 \\
& cg07320695 & 4 & 5635 & -0.24 & $6.00 \mathrm{E}-04$ & 0.26 & 0.22 \\
& cg19648967 & 14 & 2607 & -0.24 & 0.0012 & 0.72 & 0.76 \\
\hline
\end{tabular}

compared with control veterans. When we examined methylation at genes previously reported to be altered by dioxin exposure in prepubertal boys, we observed several loci with significant differences in methylation comparing the control and high exposed groups; 3 loci in the PGBD5 gene were significantly altered in the same direction as the prior study and loci in the $M B P$ and $K C N N 1$ gene loci were similarly altered in a fashion comparable to that previously reported by Pilsner et al [17]. However, none of the above findings survived correction for false discovery and thus were of limited statistical significance. At the same time, most remarkably, when we analyzed regional differences in methylation, there were numerous CpG stretches containing $\mathrm{CpGs}$ that were coordinately differentially altered after exposure, including one dramatic change in the imprinted $H 19$ region on chromosome 11 (minimum adjusted $p=10^{-28}$ ).

$H 19$ is a noncoding imprinted gene that is transcribed to form a microRNA (miR-675) [32] thought to influence early development. Neighboring H19 on the chromosome is another imprinted gene, IGF2, which is also implicated in embryogenesis. These genes are regulated by an imprinting control region that dictates the

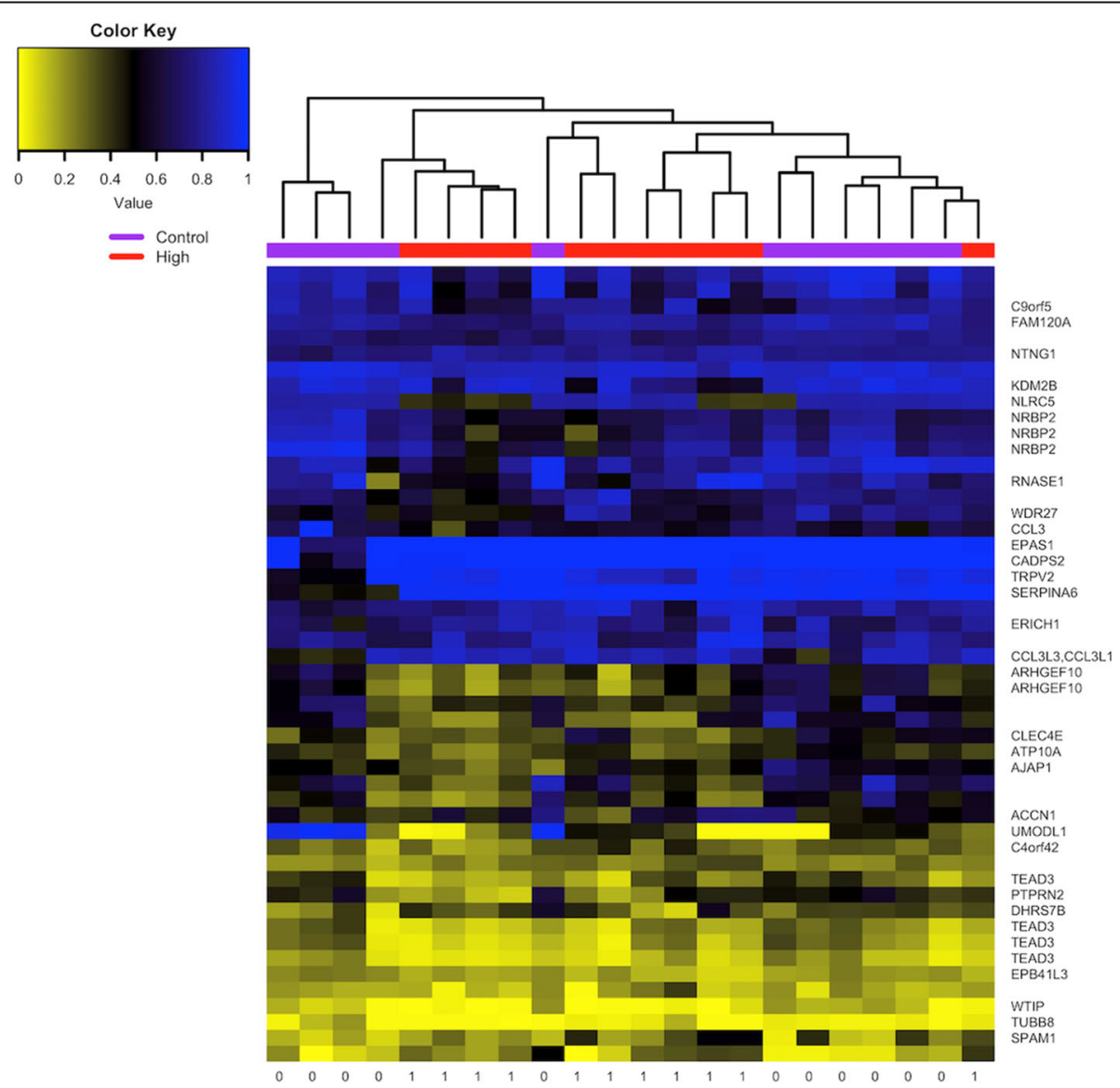

Fig. 1 Heatmap of the unstructured cluster analysis of DNA methylation in sperm of high dioxin serum level Ranch Hand veterans compared with controls 
Table 3 Mean DNA methylation in sperm by exposure group in 37 candidate CpG loci

\begin{tabular}{|c|c|c|c|c|c|c|c|}
\hline Gene & $\mathrm{CpG}$ & $\begin{array}{l}\text { Russian Low Exposure } \\
\text { Group }\end{array}$ & $\begin{array}{l}\text { Russian High Exposure } \\
\text { Group }\end{array}$ & $\begin{array}{l}\text { Ranch Hand } \\
\text { Low } \\
\text { Exposure }\end{array}$ & $\begin{array}{l}\text { Ranch Hand High } \\
\text { Exposure }\end{array}$ & $\begin{array}{l}P- \\
\text { value }\end{array}$ & $\begin{array}{l}\text { FDR adjusted } p \text { - } \\
\text { value }\end{array}$ \\
\hline SAMD11 & cg06624358 & 0.58 & 0.92 & 0.66 & 0.61 & 0.72 & 0.81 \\
\hline SAMD11 & cg14156792 & 0.51 & 0.97 & 0.60 & 0.55 & 0.77 & 0.81 \\
\hline SAMD11 & cg01727431 & 0.59 & 1 & 0.59 & 0.53 & 0.67 & 0.80 \\
\hline SAMD11 & cg02439789 & 0.46 & 0.83 & 0.58 & 0.49 & 0.51 & 0.80 \\
\hline PGBD5 & cg11570508 & 0.92 & 0.72 & 0.97 & 0.92 & 0.047 & 0.35 \\
\hline PGBD5 & $\operatorname{cg} 11226042$ & 0.97 & 0.58 & 0.93 & 0.87 & 0.039 & 0.35 \\
\hline PGBD5 & cg22875527 & 0.95 & 0.61 & 0.95 & 0.89 & 0.027 & 0.5 \\
\hline CCNY & cg05701548 & 0.14 & 0.38 & 0.57 & 0.55 & 0.77 & 0.81 \\
\hline \multirow[t]{3}{*}{ RBP3 } & cg07070043 & 0.76 & 0.34 & 0.66 & 0.56 & 0.035 & 0.35 \\
\hline & cg26066724 & 0.88 & 0.32 & 0.75 & 0.65 & 0.34 & 0.63 \\
\hline & cg07458849 & 0.91 & 0.80 & 0.88 & 0.87 & 0.87 & 0.87 \\
\hline SLC5A10 & cg04585390 & 0.60 & 0.35 & 0.58 & 0.52 & 0.34 & 0.63 \\
\hline \multirow[t]{3}{*}{$M B P$} & cg00706570 & 0.76 & 0.40 & 0.72 & 0.47 & 0.005 & 0.185 \\
\hline & cg06167221 & 0.69 & 0.80 & 0.79 & 0.69 & 0.12 & 0.40 \\
\hline & cg00593773 & 0.61 & 0.82 & 0.85 & 0.77 & 0.10 & 0.40 \\
\hline KCNN1 & cg21491340 & 0.17 & 0.07 & 0.38 & 0.30 & 0.096 & 0.40 \\
\hline KCNN1 & cg21491340 & 0.14 & 0.05 & 0.38 & 0.30 & 0.096 & 0.40 \\
\hline FBLN7 & cg11229230 & 0.83 & 0.96 & 0.98 & 0.97 & 0.20 & 0.60 \\
\hline$D G K D$ & cg24933152 & 1 & 0.92 & 0.9778 & 0.9818 & 0.060 & 0.37 \\
\hline FAM83C & cg12170299 & 0.78 & 0.72 & 0.67 & 0.63 & 0.27 & 0.63 \\
\hline FAM83C & cg11133013 & 0.858 & 0.63 & 0.73 & 0.64 & 0.21 & 0.60 \\
\hline \multirow[t]{3}{*}{ C210rf29 } & cg12061357 & 0.61 & 0.25 & 0.60 & 0.51 & 0.12 & 0.40 \\
\hline & cg26311420 & 0.56 & 0.15 & 0.40 & 0.45 & 0.66 & 0.80 \\
\hline & cg26311420 & 0.5 & 0.13 & 0.40 & 0.45 & 0.66 & 0.80 \\
\hline RBM33 & cg14353208 & 0.82 & 0.99 & 0.98 & 0.97 & 0.48 & 0.80 \\
\hline \multirow[t]{9}{*}{ RBM33 } & cg07262012 & 0.80 & 0.97 & 0.96 & 0.96 & 0.53 & 0.80 \\
\hline & cg14720951 & 0.28 & 0.14 & 0.13 & 0.17 & 0.34 & 0.63 \\
\hline & cg06182811 & 0.75 & 0.73 & 0.69 & 0.74 & 0.31 & 0.63 \\
\hline & cg06182811 & 0.85 & 0.74 & 0.69 & 0.74 & 0.31 & 0.63 \\
\hline & cg19673480 & 0.83 & 0.64 & 0.77 & 0.79 & 0.64 & 0.80 \\
\hline & cg19673480 & 0.82 & 0.59 & 0.77 & 0.79 & 0.64 & 0.80 \\
\hline & cg23541013 & 0.71 & 0.44 & 0.72 & 0.77 & 0.33 & 0.63 \\
\hline & cg01695620 & 0.74 & 0.61 & 0.83 & 0.87 & 0.43 & 0.76 \\
\hline & $\operatorname{cg} 15690180$ & 0.55 & 0.19 & 0.46 & 0.49 & 0.77 & 0.81 \\
\hline$F L 43860$ & $\operatorname{cg} 12732638$ & 0.65 & 0.84 & 0.84 & 0.85 & 0.79 & 0.81 \\
\hline PLEC1 & cg04247530 & 0.86 & 0.94 & 0.84 & 0.82 & 0.55 & 0.80 \\
\hline TBC1D2 & cg14049807 & 0.06 & 0.26 & 0.43 & 0.39 & 0.62 & 0.80 \\
\hline
\end{tabular}

expression of $H 19$ exclusively on the maternal allele, and IGF2 on the paternal allele. On the maternal side IGF2 is normally unmethylated, allowing the binding of the zinc-finger CTCF protein which blocks its expression $[6,32]$. On the other hand, H19 on the paternal side tends to be hypermethylated which enables the expression of IGF2 [33]. Interestingly, the H19 locus has previously been shown to be sensitive to alteration by TCDD; exposure to mouse preimplantation embryos causes hypermethylation and decreased expression of the $H 19$ gene [6]. However, male mice born from a mother exposed to TCDD showed no difference in 


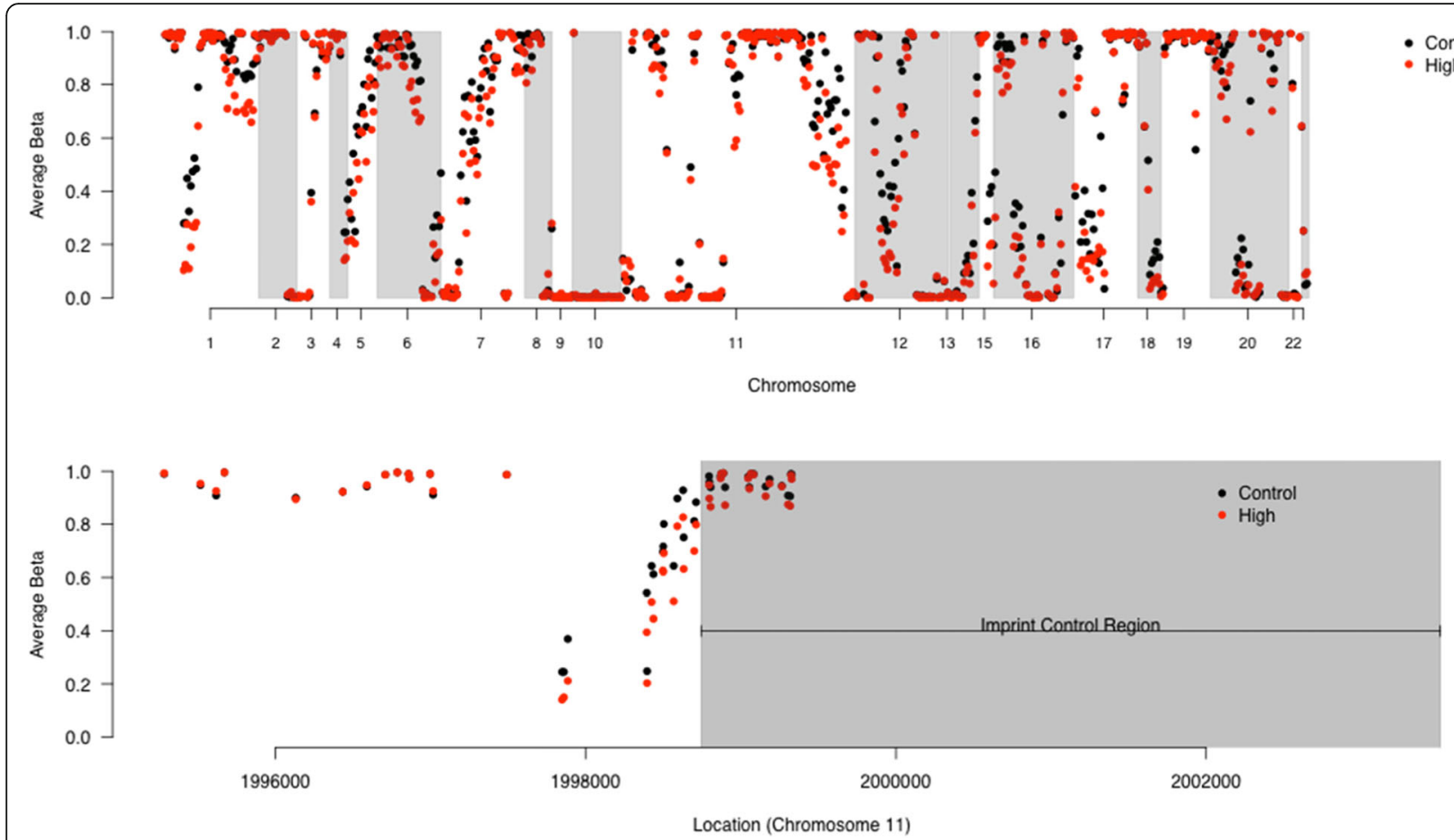

Fig. 2 Manhatten plot of the regions where alterations in DNA methylation were significantly associated with dioxin serum level

methylation in imprinted genes, but increased expression of those genes [34]. Other studies, in rodents, of reproductive toxicity have shown TCDD exposure of pregnant animals to be associated with H19-mediated cleft palate $[35,36]$, as well as with H19-mediated ovarian toxicity [37]. Studies of epigenetic alterations to the H19 gene in men have been limited; however, an investigation into the sperm epigenome of males who recurrently fathered miscarriages found the H19 ICR and CTCF6 protein binding region to be hypomethylated [32]. Hypomethylation in these loci could cause the binding of the CTCF protein on the paternal allele rather than the maternal allele, thus blocking the expression of IGF2 [32]. Similarly, the $H 19$ gene in sperm has been found to be hypomethylated in a cohort of males who practice chronic alcohol consumption [38]. Hence, while this locus seems to be clearly sensitive to DNA methylation alteration by TCDD exposure, the nature of the change (e.g. hyper vs hypomethylation, for example) may be related to the lifestage at exposure (e.g. in-utero compared with adolescent or adult exposure), to the kind of exposure (acute vs chronic) or to other parameters that remain unexplored and unknown.

Little data is available concerning the remaining gene regions found to be associated with serum dioxin level in the regional analysis. Further study of these regions would seem warranted. However, the TEAD3 gene, which we observed to be associated with TCDD level in the EWAS analysis, is a transcription factor known to interact with the YAP gene product, to participate in regulating the HIPPO pathway [39]. HIPPO signaling has been shown to be important in development of mature sperm in sheep, but its role in human sperm remains to be elucidated [40]. Hence, the significance of any association of alterations in this gene and TCDD exposure is unclear. The PGBD5 gene codes for a transposase enzyme [41] and it is possible that silencing this gene is important in maintaining the genomic stability of germ cells that undergo demethylation during development. Exposure in both studies was associated with loss of methylation at these loci. However, this finding clearly requires further investigation to fully understand its significance.

The impact of dioxin exposure on the reproductive health has been widely studied in animal models as well as exposed humans. In utero exposure to TCDD is known to decrease epididymal sperm count, fertility and testosterone levels in rats [42]. Similar exposure of mice also has been reported to induce transgenerational epigenetic alterations appreciable as changes in DNA methylation in sperm [43]. Interestingly, TCDD-exposed adult rats showed marked histological abnormalities in the testes, changes in estrogen and testosterone levels, and an altered testicular proteome [44]. Another study of adult rats exposed to TCDD showed decreased serum testosterone levels, fewer Sertoli cells, and reduced sperm production [45].

Studies in humans have produced conflicting results. Twenty-two years after a factory explosion in Seveso, 
Table 4 CpG regions (by P-Value) identified as altered in DMRcate analysis of Agent Orange exposed compared with control

\begin{tabular}{|c|c|c|c|c|c|c|c|c|c|}
\hline$\overline{\mathrm{CHR}}$ & Start & End & Width & $\begin{array}{l}\# \\
\text { CpGs }\end{array}$ & $P$-value & $\begin{array}{l}\text { Stouffer Adjusted } \\
p \text {-value }\end{array}$ & $\begin{array}{l}\text { Max beta-fold } \\
\text { change }\end{array}$ & $\begin{array}{l}\text { Mean beta-fold } \\
\text { change }\end{array}$ & $\begin{array}{l}\text { Overlapping } \\
\text { Genes }\end{array}$ \\
\hline 11 & $2,016,513$ & $2,020,560$ & 4048 & 50 & $1.83 \mathrm{E}-29$ & 1 & -0.13 & -0.04 & H19 \\
\hline 19 & $35,530,254$ & $35,532,097$ & 1844 & 9 & $5.77 \mathrm{E}-15$ & 0.93 & -0.21 & -0.15 & HPN \\
\hline 1 & $32,041,052$ & $32,042,921$ & 1870 & 12 & $8.02 \mathrm{E}-15$ & 0.99 & -0.15 & -0.08 & TINAGLI \\
\hline 20 & $62,205,530$ & $62,205,981$ & 452 & 9 & $6.21 \mathrm{E}-14$ & 0.93 & -0.12 & -0.09 & HELZ2 \\
\hline 20 & $57,581,478$ & $57,583,709$ & 2232 & 27 & $3.21 \mathrm{E}-13$ & 1 & -0.05 & -0.01 & CTSZ \\
\hline 17 & $75,315,081$ & $75,317,185$ & 2105 & 15 & $2.27 \mathrm{E}-12$ & 0.99 & -0.15 & -0.07 & SEPTO \\
\hline 11 & $2,441,045$ & $2,442,304$ & 1260 & 7 & $4.08 \mathrm{E}-12$ & 0.91 & -0.16 & -0.14 & TRPM5 \\
\hline 22 & $37,640,250$ & $37,641,506$ & 1257 & 7 & $5.82 \mathrm{E}-12$ & 0.92 & -0.11 & -0.09 & RAC2 \\
\hline 19 & $6,772,370$ & $6,773,074$ & 705 & 4 & $2.23 \mathrm{E}-11$ & 0.84 & -0.12 & -0.08 & VAV1 \\
\hline 5 & $2,007,375$ & $2,008,713$ & 1339 & 8 & $1.16 \mathrm{E}-10$ & 0.92 & -0.15 & -0.13 & $N A$ \\
\hline 16 & 725,416 & 730,187 & 4772 & 17 & $1.66 \mathrm{E}-10$ & 1 & -0.07 & -0.01 & STUB1, RHBDL1, LA16C \\
\hline 16 & $4,729,905$ & $4,733,181$ & 3277 & 14 & $1.73 \mathrm{E}-10$ & 1 & -0.2 & -0.05 & MGRN1 \\
\hline 18 & $74,691,097$ & $74,692,265$ & 1169 & 9 & $3.00 \mathrm{E}-10$ & 0.93 & -0.2 & -0.17 & $R P 11$ \\
\hline 1 & $6,129,242$ & $6,134,427$ & 5186 & 10 & $5.30 \mathrm{E}-10$ & 0.99 & 0.03 & 0 & $N A$ \\
\hline 14 & $74,823,368$ & $74,824,804$ & 1437 & 8 & 7.83E-10 & 0.92 & -0.11 & -0.07 & $N A$ \\
\hline 20 & $60,540,388$ & $60,541,082$ & 695 & 5 & $9.37 \mathrm{E}-10$ & 0.87 & -0.17 & -0.15 & $N A$ \\
\hline 6 & $169,653,612$ & $169,655,497$ & 1886 & 12 & $1.24 \mathrm{E}-09$ & 0.98 & -0.13 & -0.06 & THBS2 \\
\hline 1 & $6,085,799$ & $6,086,652$ & 854 & 9 & 1.59E-09 & 0.95 & -0.2 & -0.16 & KCNAB2 \\
\hline 2 & 495,912 & 497,296 & 1385 & 7 & $9.03 \mathrm{E}-09$ & 0.96 & -0.17 & -0.1 & $N A$ \\
\hline 12 & $133,463,607$ & $133,465,592$ & 1986 & 31 & $1.96 \mathrm{E}-08$ & 1 & 0.08 & 0.01 & CHFR, RP11 \\
\hline 11 & $2,554,198$ & $2,555,576$ & 1379 & 11 & $2.30 \mathrm{E}-08$ & 0.98 & -0.14 & -0.05 & NA \\
\hline 9 & $124,497,763$ & $124,499,870$ & 2108 & 6 & 2.73E-08 & 0.94 & -0.02 & -0.01 & NA \\
\hline 17 & $68,171,276$ & $68,171,750$ & 475 & 3 & $5.25 \mathrm{E}-08$ & 0.78 & 0.01 & 0.01 & $N A$ \\
\hline 5 & $6,775,909$ & $6,775,922$ & 14 & 2 & $2.52 \mathrm{E}-07$ & 0.76 & -0.21 & -0.2 & $N A$ \\
\hline 1 & $203,840,145$ & $203,840,808$ & 664 & 2 & 2.71E-07 & 0.76 & 0.02 & 0.01 & $N A$ \\
\hline 7 & $100,727,888$ & $100,729,412$ & 1525 & 10 & $5.27 \mathrm{E}-07$ & 0.96 & -0.1 & -0.07 & TRIM56 \\
\hline 11 & $68,778,570$ & $68,782,202$ & 3633 & 19 & $5.73 \mathrm{E}-07$ & 1 & -0.08 & -0.03 & $M R G P R F, R P 11$ \\
\hline 1 & $203,320,190$ & $203,320,541$ & 352 & 5 & $6.54 \mathrm{E}-07$ & 0.87 & -0.15 & -0.12 & FMOD \\
\hline 7 & $73,441,057$ & $73,443,113$ & 2057 & 8 & 7.83E-07 & 0.95 & -0.12 & -0.08 & ELN \\
\hline 11 & $3,186,095$ & $3,188,016$ & 1922 & 28 & 8.03E-07 & 1 & -0.09 & -0.04 & OSBPL5 \\
\hline 17 & 790,628 & 791,324 & 697 & 5 & $9.74 \mathrm{E}-07$ & 0.99 & 0.02 & 0.01 & $N A$ \\
\hline
\end{tabular}

Italy that released large amounts of TCDD, men who were exposed at prepubertal ages showed decreased sperm concentrations and motility [16]. Similarly, a prospective study of a group of Russian boys aged $8-9$ exposed to dioxins through contamination in their community found that 10 years after first exposure, higher serum TCDD concentrations were associated with decreased sperm concentrations, counts, and motility [15]. Especially in terms of sperm parameters, dioxins seem to negatively impact the reproductive health of men who were exposed at young ages. Taken together, it is thought that during the developmental stages of life, individuals are several orders of magnitude more susceptible to the effects of TCDD, resulting in pronounced consequences to fertility even at low exposures [46].

The effects of dioxin exposure on reproductive health in men exposed in puberty and adulthood is less clear. TCDD appeared to serve as a stimulant to semen production in the men from Seveso, Italy, who were exposed during puberty, and those exposed as adults showed no differences from the control group [16]. A study of men living in areas of Vietnam with high dioxin contamination found no difference in prostate-specific antigen concentrations between control and high serum dioxin groups [47]. Also, the Vietnamese men with high serum dioxin showed increased testosterone and estradiol levels, but decreased dehydroepiandrosterone levels, 
and no change in other steroid hormones [48]. Exposure to dioxin-contaminated food was associated with a decrease in total and free testosterone [49], although (as noted above) several studies have not found TCDD exposure to alter semen quality or sperm count (reviewed in [50]). Similarly, Hsu and colleagues [51] prospectively studied adult Taiwanese men exposed to dioxins through food, finding lower sperm concentrations, increased incidence of abnormal sperm morphology, and reduced ability of spermatozoa to penetrate hamster oocytes. We studied veterans who were young adults at the time of exposure (in their 20s) and thus may have been at the limits of or beyond any putative 'window of susceptibility' to the induction of epigenetic changes by dioxin. As noted above, the veterans that were studied here did not demonstrate any changes in semen analysis, specifically including sperm count and quality.

This work has several limitations. Perhaps most importantly, we were quite underpowered to find epigenome-wide alterations in sperm associated with AO exposure, since we studied only 37 veterans. Additionally, while TCDD exposure occurred from 1962 to 1971 , participants were not initially studied until 1982 [19]. Given that the half-life of TCDD in humans is 7 to 11 years [1], the measured serum dioxin levels were lower than those incurred during the exposure period. This, along with measurement error associated with the device/instrument used to measure serum dioxin levels, also could have affected our ability to detect induced alterations. While we attempted to adjust for all compounding factors, the latency of our analysis following the initial exposure allowed for a large window of time for uncontrolled factors to make an impact. Although we used a genome-wide platform for assessing DNA methylation, it was limited in its interrogation of loci studied using different methods (e.g. Pilsner et al [17] who used RRBS).

In summary, studying a small number of sperm samples from AO-exposed Ranch Hand veterans enrolled in the AFHS, we did not find evidence of significant epigenome-wide alterations associated with AO exposure. However, we did replicate several of the findings of a prior study of dioxin-exposed Russian boys in an analysis restricted to study of overlapping loci on the 2 platforms. Additionally, we produced evidence that the H19 gene region, among others, is altered in the sperm of Ranch Hand veterans. Due to the importance of this regions association with adverse reproductive outcomes and the suggestion of other identified regions to be similarly impacted by Agent Orange exposure, further investigation is warranted.

\section{Supplementary information}

Supplementary information accompanies this paper at https://doi.org/10. 1186/s12940-019-0533-z.

Additional file 1: Figure S1. Volcano plot of the comparison of CpG-specific methylation values and dioxin exposure.

Abbreviation

AFHS: Air Force Health Study

\section{Acknowledgements \\ The authors acknowledge the kind assistance of Dr. Anne Styka and Dr. David Butler of the National Academy of Sciences.}

\section{Authors' contributions}

Drs. Kelsey and Boekelheide conceived of the research. Drs. Houseman, Dere, Huse, Koestler and Eliot conducted the analysis. Dr. Butler conducted the laboratory work. Mr. Rytel drafted the manuscript with assistance of Dr. Kelsey and all of the co-authors. All authors read and approved the final manuscript.

\section{Funding}

This work was funded in part by a grant from the National Academy of Sciences.

Availability of data and materials

The data are available on GEO (pending acceptance).

Ethics approval and consent to participate

This research was approved by the Brown University IRB.

Consent for publication

All of the authors have reviewed and approved the manuscript for publication.

\section{Competing interests}

Dr. Karl Kelsey is a founder and advisor for Cellintec. The other authors declare they have no actual or potential competing financial interests.

\section{Author details}

${ }^{1}$ Department of Epidemiology, Brown University School of Public Health, Providence, RI 02912, USA. ²Department of Pathology and Laboratory Medicine, Brown University School of Public Health, Providence, RI 02912, USA. ${ }^{3}$ NIAID Collaborative Bioinformatics Resource, Frederick National Laboratory for Cancer Research, Frederick, MD 21701, USA. ${ }^{4}$ La Center, USA. ${ }^{5}$ Department of Biostatistics \& Data Science, University of Kansas Medical Center, Kansas City, KS 66160, USA.

Received: 3 July 2019 Accepted: 1 October 2019

Published online: 29 October 2019

\section{References}

1. Veterans and Agent OrangeHealth Effects of Herbicides Used in Vietnam. Institute of Medicine (US) Committee to Review the Health Effects in Vietnam Veterans of Exposure to Herbicides.Washington (DC): National Academies Press (US); 1994. ISBN-10: 0-309-04887-7.

2. National Academies of Sciences, Engineering, and Medicine. Veterans and Agent Orange: Update 11 (2018). Washington, DC: The National Academies Press; 2018. https://doi.org/10.17226/25137.

3. Mrema EJ, et al. Persistent organochlorinated pesticides and mechanisms of their toxicity. Toxicology. 2013;307:74-88.

4. Patrizi B, Siciliani de Cumis M. TCDD Toxicity Mediated by Epigenetic Mechanisms. Int J Mol Sci. 2018;19(12):4101. https://doi.org/10.3390/ ijms19124101Review.

5. Singh NP, et al. Activation of aryl hydrocarbon receptor (AhR) leads to reciprocal epigenetic regulation of FoxP3 and IL-17 expression and amelioration of experimental colitis. PLoS One. 2011;6(8):e23522.

6. $\mathrm{Wu} \mathrm{Q}$, et al. Exposure of mouse preimplantation embryos to 2,3,7,8tetrachlorodibenzo-p-dioxin (TCDD) alters the methylation status of imprinted genes H19 and Igf2. Biol Reprod. 2004;70(6):1790-7. 
7. Wang $C$, et al. Preliminary research on DNA methylation changes during murine palatogenesis induced by TCDD. J Craniomaxillofac Surg. 2017:45(5):678-84

8. Olsvik PA, et al. Impacts of TCDD and MeHg on DNA methylation in zebrafish (Danio rerio) across two generations. Comp Biochem Physiol C Toxicol Pharmacol. 2014;165:17-27.

9. Aluru N, Jenny MJ, Hahn ME. Knockdown of a zebrafish aryl hydrocarbon receptor repressor (AHRRa) affects expression of genes related to photoreceptor development and hematopoiesis. Toxicol Sci. 2014;139(2):381-95.

10. Pilsner JR, et al. Spermatogenesis disruption by dioxins: epigenetic reprograming and windows of susceptibility. Reprod Toxicol. 2017;69:221-9.

11. Iqbal K, et al. Deleterious effects of endocrine disruptors are corrected in the mammalian germline by epigenome reprogramming. Genome Biol. 2015;16:59.

12. Anway MD, Leathers C, Skinner MK. Endocrine disruptor vinclozolin induced epigenetic transgenerational adult-onset disease. Endocrinology. 2006; 147(12):5515-23.

13. Guerrero-Bosagna C, et al. Epigenetic transgenerational actions of vinclozolin on promoter regions of the sperm epigenome. PLoS One. 2010;5(9). https:// doi.org/10.1371/journal.pone.0013100.

14. Guerrero-Bosagna C, et al. Epigenetic transgenerational inheritance of vinclozolin induced mouse adult onset disease and associated sperm epigenome biomarkers. Reprod Toxicol. 2012;34(4):694-707.

15. Minguez-Alarcon $L$, et al. A longitudinal study of Peripubertal serum Organochlorine concentrations and semen parameters in young men: the Russian Children's study. Environ Health Perspect. 2017;125(3):460-6.

16. Mocarelli $\mathrm{P}$, et al. Dioxin exposure, from infancy through puberty, produces endocrine disruption and affects human semen quality. Environ Health Perspect. 2008;116(1):70-7.

17. Pilsner JR, et al. Peripubertal serum dioxin concentrations and subsequent sperm methylome profiles of young Russian adults. Reprod Toxicol. 2018;78:40-9.

18. Henriksen GL, Michalek JE. Serum dioxin, testosterone, and gonadotropins in veterans of operation ranch hand. Epidemiology. 1996;7(4):454-5.

19. The Air Force Health Study Assets Research Program. Committee on the Management of the Air Force Health Study Data and Specimens-Report to Congress; Board on the Health of Select Populations; Institute of Medicine. Washington (DC): National Academies Press (US); 2015.

20. Patterson DG Jr, et al. High-resolution gas chromatographic/high-resolution mass spectrometric analysis of human serum on a whole-weight and lipid basis for 2,3,7,8-tetrachlorodibenzo-p-dioxin. Anal Chem. 1987;59(15):2000-5

21. CDC. Health Status of Vietnam Veterans: Supplement A, Laboratory Methods and Quality Control; 1989. p. 195-204.

22. Bianchi E, et al. Spermatozoal large RNA content is associated with semen characteristics, sociodemographic and lifestyle factors. PLoS One. 2019:14(5):e0216584.

23. Lathrop G, Wolfe WH, Albanese RA, Moynahan PM. Epidemiologic investigation of health effects in air force personnel following exposure to herbicides: Baseline morbidity study results. San Antonio: USAF School of aerospace medicine: brooks AFB; 1984.

24. Wilhelm-Benartzi CS, et al. Review of processing and analysis methods for DNA methylation array data. Br J Cancer. 2013:109(6):1394-402.

25. Chen YA, et al. Discovery of cross-reactive probes and polymorphic CpGs in the Illumina Infinium HumanMethylation450 microarray. Epigenetics. 2013;8(2):203-9.

26. Triche TJ Jr, et al. Low-level processing of Illumina Infinium DNA methylation BeadArrays. Nucleic Acids Res. 2013;41(7):e90

27. Fortin JP, et al. Functional normalization of $450 \mathrm{k}$ methylation array data improves replication in large cancer studies. Genome Biol. 2014;15(12):503.

28. Teschendorff $A E$, et al. A beta-mixture quantile normalization method for correcting probe design bias in Illumina Infinium 450 k DNA methylation data. Bioinformatics. 2013;29(2):189-96.

29. Leek JT, Storey JD. Capturing heterogeneity in gene expression studies by surrogate variable analysis. PLoS Genet. 2007;3(9):1724-35.

30. Mallik S, Odom GJ, Gao Z, Gomez L, Chen X, Wang L. An Evaluation of Supervised Methods for Identifying Differentially Methylated Regions in Illumina Methylation Arrays. Brief Bioinform. 2019. https:/doi.org/10.1093/bib/bby085.

31. Riley JW, S S, Suchman EA, Devinney LC, Star SA, Williams RM. The American Soldier: Adjustment During Army Life. Princeton: Princeton University Press; 1949
32. Ankolkar M, et al. Methylation analysis of idiopathic recurrent spontaneous miscarriage cases reveals aberrant imprinting at H19 ICR in normozoospermic individuals. Fertil Steril. 2012;98(5):1186-92.

33. Thorvaldsen JL, Duran KL, Bartolomei MS. Deletion of the $\mathrm{H} 19$ differentially methylated domain results in loss of imprinted expression of H19 and lgf2. Genes Dev. 1998;12(23):3693-702.

34. Somm E, Stouder C, Paoloni-Giacobino A. Effect of developmental dioxin exposure on methylation and expression of specific imprinted genes in mice. Reprod Toxicol. 2013;35:150-5.

35. Gao LY, et al. LncRNA H19 and target gene-mediated cleft palate induced by TCDD. Biomed Environ Sci. 2017;30(9):676-80.

36. Gao L, et al. LncRNA H19-mediated mouse cleft palate induced by all-trans retinoic acid. Hum Exp Toxicol. 2017:36(4):395-401.

37. Zhang $X$, et al. Role of epigenetic regulation of Igf2 and H19 in 2,3,7,8Tetrachlorobenzo-p-dioxin (TCDD)-induced ovarian toxicity in offspring rats. Toxicol Lett. 2019;311:98-104.

38. Ouko LA, et al. Effect of alcohol consumption on $\mathrm{CpG}$ methylation in the differentially methylated regions of H19 and IG-DMR in male gametes: implications for fetal alcohol spectrum disorders. Alcohol Clin Exp Res. 2009;33(9):1615-27.

39. Noland $\mathrm{CL}$, et al. Palmitoylation of TEAD transcription factors is required for their stability and function in Hippo pathway signaling. Structure. 2016;24(1):179-86

40. Zhang GM, et al. Expression of Hippo signaling pathway components in $\mathrm{Hu}$ sheep male reproductive tract and spermatozoa. Theriogenology. 2019;126:239-48.

41. Henssen AG, Henaff E, Jiang E, Eisenberg AR, Carson JR, Villasante CM, Ray M, Still E, Burns M, Gandara J, Feschotte C, Mason CE, Kentsis A. Genomic DNA transposition induced by human PGBD5. eLife. 2015;4:e10565. PMCID: PMC4625184. Published online 2015 Sep 25. https://doi.org/10.7554/ eLife.10565.

42. Foster WG, Maharaj-Briceno S, Cyr DG. Dioxin-induced changes in epididymal sperm count and spermatogenesis. Cien Saude Colet. 2011; 16(6):2893-905.

43. Manikkam M, et al. Dioxin (TCDD) induces epigenetic transgenerational inheritance of adult onset disease and sperm epimutations. PLoS One. 2012; 7(9):e46249.

44. Choi JS, et al. Effect of 2,3,7,8-tetrachlorodibenzo-p-dioxin on testicular spermatogenesis-related panels and serum sex hormone levels in rats. BJU Int. 2008;101(2):250-5

45. Simanainen $\mathrm{U}$, et al. Adult 2,3,7,8-tetrachlorodibenzo-p-dioxin (TCDD) exposure and effects on male reproductive organs in three differentially TCDD-susceptible rat lines. Toxicol Sci. 2004;81(2):401-7.

46. Peterson RE, Theobald HM, Kimmel GL. Developmental and reproductive toxicity of dioxins and related compounds: cross-species comparisons. Crit Rev Toxicol. 1993;23(3):283-335.

47. Sun $X$, et al. The relationship between agent Orange and prostate specific antigen: a comparison of a hotspot and a non-sprayed area in Vietnam. Environ Health Prev Med. 2013;18(5):356-60.

48. Sun $\mathrm{XL}$, et al. Influence of dioxin exposure upon levels of prostate-specific antigen and steroid hormones in Vietnamese men. Environ Sci Pollut Res Int. 2016;23(8):7807-13.

49. Dhooge $W$, et al. Serum dioxin-like activity is associated with reproductive parameters in young men from the general Flemish population. Environ Health Perspect. 2006;114(11):1670-6.

50. Toft G. Persistent organochlorine pollutants and human reproductive health Dan Med J. 2014;61(11):B4967.

51. Hsu PC, et al. Sperm changes in men exposed to polychlorinated biphenyls and dibenzofurans. JAMA. 2003;289(22):2943-4.

\section{Publisher's Note}

Springer Nature remains neutral with regard to jurisdictional claims in published maps and institutional affiliations. 